[Article]

www.whxb.pku.edu.cn

\title{
$\mathrm{CdS} /$ 石墨烯复合材料的制备及其可见光催化分解水产氢性能
}

\author{
敏世雄吕功煊 \\ (中国科学院兰州化学物理研究所, 羰基合成与选择氧化国家重点实验室, 兰州 730000)
}

\begin{abstract}
摘要: 以氧化石墨烯和 CdS 为原料, 在乙醇水溶液中采用 CdS 光催化还原法制备了 CdS/石墨烯复合光催化 材料, 并用透射电子显微镜 (TEM)、X射线衍射 $(X R D)$ 、傅里叶变换红外 (FTIR)光谱、X射线光电子能谱(XPS)和 瞬态光电流等技术对复合材料的结构和光电性能进行了表征. 可见光照射下 $(\lambda \geq 420 \mathrm{~nm})$, 研究了该复合材料光 催化分解水产氢的性能. 结果表明, 可见光照射下 CdS 的光生电子可有效地还原氧化石墨烯, 得到 CdS 与石墨 烯之间具有强相互作用力的 CdS/石墨烯复合材料. 与 CdS 相比, 复合材料中石墨烯作为良好的电子受体和传 递介质, 可明显加快 $\mathrm{CdS}$ 光生电子的迁移速率, 提高光生载流子的分离效率, 从而增强复合材料的光电性能和 光催化分解水产氢的活性.
\end{abstract}

关键词: CdS; 石墨烯; 光电性能; 产氢; 可见光

中图分类号: 0643.32

\section{Preparation of CdS/Graphene Composites and Photocatalytic Hydrogen Generation from Water under Visible Light Irradiation}

\author{
MIN Shi-Xiong LÜ Gong-Xuan* \\ (State Key Laboratory for Oxo Synthesis and Selective Oxidation, Lanzhou Institute of Chemical Physics, \\ Chinese Academy of Sciences, Lanzhou 730000, P. R. China)
}

\begin{abstract}
CdS/graphene composite photocatalysts were prepared by photocatalytically reducing graphene oxide with CdS nanoparticles in an aqueous ethanol solution. The structure and photoelectrical properties of the resulted materials were characterized by transmission electron microscopy (TEM), X-ray diffraction (XRD), Fourier transform infrared (FTIR) spectroscopy, X-ray photoelectron spectroscopy (XPS), and transient photocurrent measurements. The photocatalytic activities of the CdS/graphene composites for hydrogen evolution were evaluated under visible light irradiation $(\lambda \geq 420 \mathrm{~nm})$. The results show that the graphene oxide can be efficiently reduced by the photogenerated electrons of $\mathrm{CdS}$ and thus $\mathrm{CdS} /$ graphene composite is formed and it shows strong interactions between CdS and graphene. Compared with CdS, the enhanced photocurrent generation and photocatalytic activity toward hydrogen evolution for the CdS/ graphene composite photocatalysts could be attributed to the ability of graphene to capture and transport electrons, and to promote charge separation.
\end{abstract}

Key Words: CdS; Graphene; Photoelectrical property; Hydrogen generation; Visible light

Received: April 22, 2011; Revised: June 27, 2011; Published on Web: July 4, 2011.

"Corresponding author. Email: gxlu@lzb.ac.cn; Tel: +86-931-4968178.

The project was supported by the National Key Basic Research Program of China (973) (2007CB613305, 2009CB22003), National High-Tech Research and Development Program of China (863) (2009AA05Z117) and Solar Energy Project of Chinese Academy of Sciences

(KGCX2-YW-390-1, KGCX2-YW-390-3).

国家重点基础研究发展规划项目(973) (2007CB613305, 2009CB22003), 国家高技术研究发展计划项目(863) (2009AA05Z117)和中国科学院太

阳能行动计划(KGCX2-YW-390-1, KGCX2-YW-390-3)资助

(C) Editorial office of Acta Physico-Chimica Sinica 


\section{1 引 言}

石墨烯 (graphene)是新型二维碳纳米材料, ${ }^{1,2}$ 其 能隙为零, 与碳纳米管 $(\mathrm{CNTs})$ 和富勒烯 $\left(\mathrm{C}_{60}\right)$ 相比, 石 墨烯有更大的比表面积 $\left(2630 \mathrm{~m}^{2} \cdot \mathrm{g}^{-1}\right) 、{ }^{3}$ 高化学稳定 性以及更为优异的电子传导性能 $\left(2 \times 10^{5} \mathrm{~cm}^{2} \cdot \mathrm{V}^{-1}\right.$. $\left.\mathrm{s}^{-1}\right){ }^{4}$ 研究表明, 石墨烯可以作为电子传递介质提高 半导体中光生电子的迁移速率, 降低载流子的复合 几率, 提高太阳能电池的光电转化效率. ${ }^{5-8}$ 将石墨烯 与半导体光催化剂复合, 利用石墨烯规整的二维平 面结构作为光催化剂的载体, 一方面可以提高催化 剂的分散程度, 另一方面可加快光生电荷迁移的速 率, 提高复合材料的光催化活性. ${ }^{9-13}$ Zhang 等 ${ }^{9}$ 采用 水热法合成了 P25-石墨烯(P25-G)复合材料, 发现石 墨烯的引入不仅能增强复合材料对亚甲基蓝的吸 附性能, 而且将光响应范围拓展至可见光区; 同时, 石墨烯作为电子受体抑制了复合体系光生电子-空 穴的复合, 从而增强了复合材料的光催化性能, 使 其表现出优于 P25-CNTs 的光催化性能. Kamat 等 ${ }^{14-16}$ 对 $\mathrm{TiO}_{2}$ 与石墨烯间的电子传递机制进行了系 统的研究, 进一步证实了石墨烯作为电子 “受体” 储 存电子和作为 “导线”转移电子的功能.

在半导体光电转化和光催化过程中, 促进半导 体光生电子-空穴分离是石墨烯的主要作用, 该作用 不仅在光电转化、光催化降解污染物方面发挥着重 要的作用, 也将在光解水制氢的研究中得到广泛的 应用. 然而, 将石墨烯应用于光催化制氢中的研究 报道较少. Zhang 等 ${ }^{17}$ 采用溶胶-凝胶法制备了 $\mathrm{TiO}_{2} /$ 石墨烯复合材料, 其在紫外-可见光条件下光解水制 氢活性是 P25 的 2 倍. $\mathrm{Ng}$ 等 ${ }^{18}$ 采用光催化还原法制 备了 $\mathrm{BiVO}_{4}$ /石墨烯光催化材料, 在外加偏压条件下 $(0.8 \mathrm{~V})$, 以 $\mathrm{BiVO}_{4} /$ 石墨烯为光电极时, 光电池可稳定 地分解水产生 $\mathrm{H}_{2}$ 和 $\mathrm{O}_{2}$, 而以 $\mathrm{BiVO}_{4}$ 为光电极时, 无 $\mathrm{H}_{2}$ 和 $\mathrm{O}_{2}$ 产生. 光电化学性能的研究表明, 石墨烯 的加入不仅提高了激发 $\mathrm{BiVO}_{4}$ 所产生电子的寿命, 降低了光生电子-空穴的复合几率, 而且也增加了 $\mathrm{BiVO}_{4}$ 与导电基底的接触面积, 从而提高了光电池 光电转化和光催化反应的效率. 但以上研究的体系 只能在紫外光下或外加电场下工作, 从利用太阳能 的角度出发, 开发可见光响应的半导体/石墨烯复合 光催化材料将具有更大的应用前景. 本文以氧化石 墨烯分散液和 $\mathrm{CdS}$ 为反应原料, 在可见光条件下, 利用 $\mathrm{CdS}$ 光催化还原的方法制备了 $\mathrm{CdS} /$ 石墨烯复 合材料. 利用多种技术手段对复合材料的结构和光
电性能进行了表征, 研究了复合材料可见光下光催 化分解水产氢性能, 并与 $\mathrm{CdS}$ 进行了对比分析, 期 望通过石墨烯的引入提高复合材料光生载流子的 分离效率, 增强 $\mathrm{CdS}$ 在可见光下光催化分解水产氢 的效率.

\section{2 实验部分}

\section{1 试剂和仪器}

$\mathrm{Cd}\left(\mathrm{CH}_{3} \mathrm{COOH}\right)_{2} \cdot 2 \mathrm{H}_{2} \mathrm{O}$ (分析纯, 国药集团化学 试剂有限公司); $\mathrm{Na}_{2} \mathrm{~S} \cdot 9 \mathrm{H}_{2} \mathrm{O}$ (分析纯, 成都市科龙化 工试剂厂); 石墨粉(化学纯, 国药集团化学试剂有限 公司); $\mathrm{NaNO}_{3}$ (分析纯, 国药集团化学试剂有限公 司), $\mathrm{P}_{2} \mathrm{O}_{5}$ (分析纯, 成都市科龙化工试剂厂); $\mathrm{KMnO}_{4}$ (分析纯, 北京化工厂) $\mathrm{H}_{2} \mathrm{O}_{2}$ (分析纯, 30\%, 天津市 红岩化学试剂试剂厂); 浓硫酸(分析纯, 95\%-98\%, 白银市良友化学试剂有限公司); 浓 $\mathrm{HCl}$ (分析纯, $36 \%-38 \%$, 白银市良友化学试剂有限公司); 实验用 水为蒸馏水. 高分辨透射电子显微镜(HRTEM)表征 在 Tecnai F-30FEG 型高分辨透射电镜上进行(FEI 公 司, 美国), 加速电压为 $300 \mathrm{kV}$; 采用 ESCALAB210XPS 光电子能谱仪(VG Scientific 公司, 英国)分析和 表征催化剂化学组成和化学状态 $(\mathrm{Mg}$ 靶, C $1 s$ 校正 到 $285.00 \mathrm{eV}) ; \mathrm{XRD}$ 晶相分析在 $\mathrm{D} / \mathrm{max} \mathrm{RB}$ 型 $\mathrm{X}$ 射线 粉末衍射仪(日本电子公司, 日本)上进行 $\left(\mathrm{Cu} K_{a}\right.$, 管 电流 $30 \mathrm{~mA}$, 管电压 $40 \mathrm{kV}$ ); 红外光谱在 Nexus 870 型红外光谱仪(Nicolet 公司, 美国)上测定, $\mathrm{KBr}$ 压 片.

\section{2 光催化还原法制备 CdS/石墨烯复合光催化剂}

氧化石墨(GO)采用改进的 Hummers 法制备; $;^{19,20}$ $\mathrm{CdS}$ 采用化学沉淀-水热晶化法合成. ${ }^{21} \mathrm{CdS} /$ 石墨烯 复合材料采用光催化还原法合成: 将一定量的氧化 石墨分散到 $100 \mathrm{~mL} \mathrm{20 \%}$ 的乙醇水溶液中, 超声分散 $(200 \mathrm{~W}, 25 \mathrm{kHz})$ 得到均一氧化石墨烯分散液 (0.005-0.25 mg $\left.\cdot \mathrm{L}^{-1}\right)$. 将该分散液转移至 $150 \mathrm{~mL}$ 带 平面窗口的石英反应瓶中, 加入 $0.5 \mathrm{~g} \mathrm{CdS}$ 纳米粒 子, 超声分散 $0.5 \mathrm{~h}$, 高纯 $\mathrm{Ar}$ 气吹扫 $1 \mathrm{~h}$, 然后置于 $300 \mathrm{~W}$ 碘铇灯下(用 $420 \mathrm{~nm}$ 的截至滤光片滤去紫外 光)光催化还原 $3 \mathrm{~h}$, 产物离心分离, 依次用蒸馏水和 无水乙醇洗涤, $60{ }^{\circ} \mathrm{C}$ 干燥, 研磨备用. 采用以上方 法, 改变制备过程中氧化石墨烯分散液的浓度, 得 到一系列不同石墨烯含量的 CdS/石墨烯复合材料, 记为 $\mathrm{CdS} /$ graphene $(w)$, 其中 $w$ 为复合材料中石墨烯 的质量分数. 


\section{3 光催化分解水产氢实验}

光催化分解水产氢实验在带平面窗口的石英 反应瓶 $(150 \mathrm{~mL})$ 中进行, 光源为 $300 \mathrm{~W}$ 碘铇灯, 配有 $420 \mathrm{~nm}$ 截至滤光片. 将 $50 \mathrm{mg}$ 催化剂加入到 $100 \mathrm{~mL}$ $0.1 \mathrm{~mol} \cdot \mathrm{L}^{-1} \mathrm{Na}_{2} \mathrm{~S}$ 和 $0.1 \mathrm{~mol} \cdot \mathrm{L}^{-1} \mathrm{Na}_{2} \mathrm{SO}_{3}$ 的混合溶 液中, 加入一定量的 $\mathrm{H}_{2} \mathrm{PtCl}_{6}$ 溶液, 原位光沉积载铂 (质量分数为 $0.5 \%$ ). 光照前体系超声分散 $5 \mathrm{~min}$, 通 氩气置换 $40 \mathrm{~min}$, 光照过程中用磁力搅拌器搅拌, 使 催化剂保持悬浮状态, 每隔一定时间从反应瓶的气 相中抽取 $0.5 \mathrm{~mL}$ 的样品, 在 Agilent 6820 (Agilent 公 司, 美国)气相色谱仪上分析产生的 $\mathrm{H}_{2}$ 量(热导检测 器, 载气为氞气, $13 X$ 分子篮填充柱), 外标法定量.

\section{4 工作电极的制备和光电性能测试}

工作电极采用物理沉积法制备: 导电玻璃(ITO) 依次在溶有表面活性剂的蒸馏水、稀 $\mathrm{HCl}$ 和稀 $\mathrm{NaOH}$ 溶液中超声清洗, 最后用蒸馏水冲洗, 在 $80^{\circ} \mathrm{C}$ 干燥. 取一定量的 $\mathrm{CdS}$ 和 $\mathrm{CdS} /$ 石墨烯 (w) 粉末超声分 散在蒸馏水中形成悬浮液 $\left(2 \mathrm{~g} \cdot \mathrm{L}^{-1}\right)$. 用微量注射器 抽取一定体积的悬浮液, 滴加于 ITO 玻璃表面, 在红 外灯下缓慢干燥, 得到负载有催化剂的 ITO工作电 极, 所制备的工作电极接触电解质和光辐射的几何 面积约为 $1.6 \mathrm{~cm}^{2}$. 光电化学实验在 CHI660A (上海 辰华仪器公司) 电化学工作站上进行, 对电极为 $\mathrm{Pt}$ 片
电极, 参比电极为饱和甘录电极 (SCE), 电解液为 $0.1 \mathrm{~mol} \cdot \mathrm{L}^{-1} \mathrm{Na}_{2} \mathrm{SO}_{4}$ 水溶液, 光源为 $250 \mathrm{~W}$ 碘铇灯, 用 $420 \mathrm{~nm}$ 的截止滤光片滤去紫外光.

\section{3 结果和讨论}

\section{$3.1 \mathrm{CdS} /$ 石墨烯复合光催化剂的制备}

$\mathrm{CdS} /$ 石墨烯复合光催化剂制备过程如图 1 所 示. 首先采用改进的 Hummers 法制备了氧化石墨 $(\mathrm{GO})$, 在乙醇水溶液中超声剥离 $\mathrm{GO}$ 得到稳定的氧 化石墨烯分散液. 向其中加入 $\mathrm{CdS}$ 纳米粒子, 在脱 氧条件下, 可见光照激发 $\mathrm{CdS}$ 分别在导带和价带产 生光生电子和空穴, 在价带空穴被牺牲试剂 $\mathrm{C}_{2} \mathrm{H}_{5} \mathrm{OH}$ 所消耗的同时(图 1a), 累积在 $\mathrm{CdS}$ 导带上的 光生电子向氧化石墨烯迁移, ${ }^{14,15}$ 由于其强的还原 性, 还原氧化石墨烯上的含氧基团得到了 $\mathrm{CdS} /$ 石墨 烯复合材料(图 1b). CdS 纳米粒子分散到氧化石墨 烯分散液中, 光照前溶液的颜色为黄色; 光照过程 中, 分散体系的颜色由黄色向黄绿色转变, 并随着 光照时间的延长, 颜色逐渐加深(图 2(a, b)), 这与 $\mathrm{Ng}$ 等 ${ }^{18}$ 在制备 $\mathrm{BiVO}_{4} /$ 石墨烯复合材料过程中观察到的 现象一致, 表明氧化石墨烯被光激发 $\mathrm{CdS}$ 产生的电 子还原生成了石墨烯, 形成 $\mathrm{CdS}$ /石墨烯复合材料. 另外, 在光催化还原过程中, 分散体系的颜色均一,

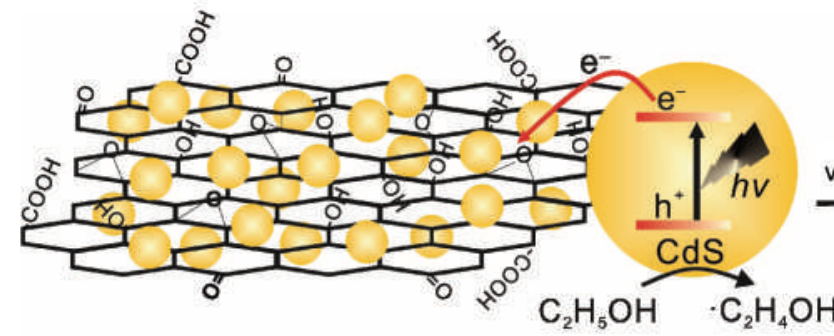

graphene oxide sheets

CdS nanoparticles

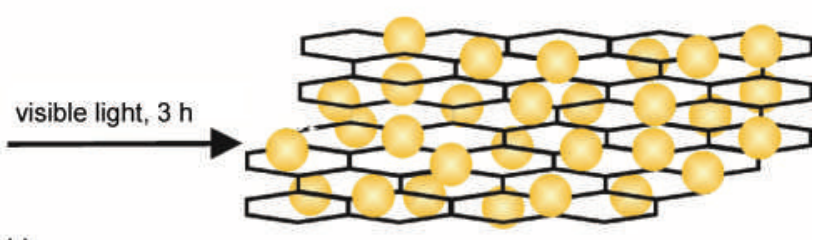

CdS/graphene composites

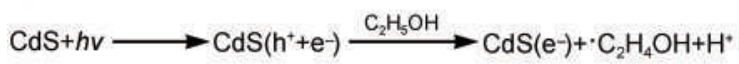

(a) $\mathrm{CdS}\left(\mathrm{e}^{-}\right)+$graphene oxide CdS+graphene

(b)

图 1 光催化还原法制备 $\mathrm{CdS} /$ 石墨烯复合材料的示意图

Fig.1 Schematic diagram of synthesis of $\mathrm{CdS} /$ graphene composites using photocatalytic reduction
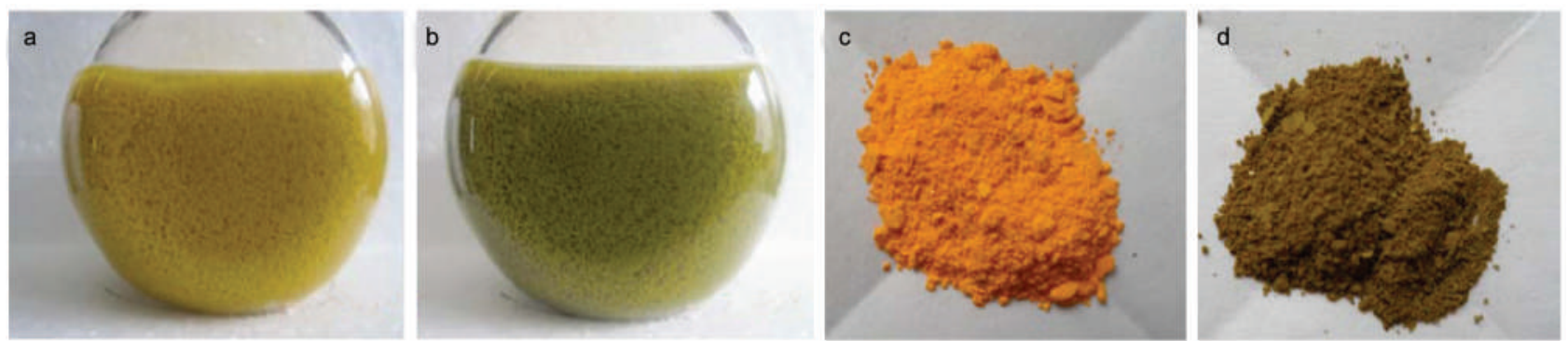

图 2 氧化石墨烯和 CdS 悬浮液光照前(a)、后(b)和 CdS (c)、CdS/石墨烯(d)粉末的照片

Fig.2 Photos of graphene oxide and CdS suspension in aqueous ethanol solution before visible light irradiation (a) and after $3 \mathrm{~h}$ of visible light irradiation (b), CdS (c) and CdS/graphene (d) powders 
干燥之后(图 2(c,d)), 没有观察到相分离现象的发 生, 说明在光催化还原过程中石墨烯与 $\mathrm{CdS}$ 之间存 在较强的作用力, 这种强作用力的存在有利于复合 体系电荷的分离和迁移, 增强光催化性能.

\section{$3.2 \mathrm{CdS} /$ 石墨烯复合光催化剂的结构表征}

图 3 为复合材料的 TEM 和 HRTEM 照片. 从图 3a中可明显观察到在复合材料中二维石墨烯片层 穿插于 $\mathrm{CdS}$ 纳米粒子之间, 作为电荷传递的介质将 $\mathrm{CdS}$ 纳米粒子彼此链接起来形成 $\mathrm{CdS} /$ 石墨烯复合 材料. 这种结构与文献 ${ }^{9,13,15}$ 报道的石墨烯负载光催 化剂的结构明显不同, 可能是复合材料中石墨烯的 含量较少和制备方法的差异导致的. 与负载型催化 剂相比, 石墨烯片层在半导体纳米粒子之间的穿插 可能更有利于光生电荷的传递, 提高载流子的分离 效率(图 3a 插图). ${ }^{18}$ 从复合材料的高分辨电镜照片 (图 3b)中可以看到, 石墨烯的边沿部位卷曲成褶皱 状, 是单层或少数层石墨烯的特征. $\mathrm{CdS}$ 纳米粒子为 球形颗粒, 形貌均一, 平均粒径约为 28-35 nm, 其高 分辨电镜可明显观察到清晰完整的晶格条纹(图 3b 插图), 说明 $\mathrm{CdS}$ 结晶良好, 条纹间距约为 $0.330 \mathrm{~nm}$, 与纤锌矿结构 $\mathrm{CdS}(002)$ 晶面间距相吻合, 表明复合 材料中 $\mathrm{CdS}$ 具有六方纤锌矿结构.

图 4a 为石墨粉和由 Hummers 法制备的氧化石 墨的XRD 谱图. 石墨粉在 $26.58^{\circ}$ 处出现了(002)晶面 的特征衍射峰, 对应的层间距为 $0.335 \mathrm{~nm}$. 经氧化 后, 石墨的(002)特征衍射峰消失, 而在 $10.68^{\circ}$ 处出现 了氧化石墨(001)的特征衍射峰, 层间距增大至 $0.828 \mathrm{~nm}$, 表明氧化使石墨层间键合了大量含氧官 能团, 形成氧化石墨. ${ }^{22}$ 图 $4 \mathrm{~b}$ 为 $\mathrm{CdS}$ 和 $\mathrm{CdS} /$ 石墨烯复 合材料的 XRD 谱图. $\mathrm{CdS}$ 在 $2 \theta$ 为 $24.86^{\circ} 、 26.53^{\circ}$ 、 $28.22^{\circ} 、 36.74^{\circ} 、 43.84^{\circ} 、 47.89^{\circ} 、 51.94^{\circ}$ 处出现衍射峰, 与 CdS 标准谱图一致(PDF No. 77-2306), 表明所制
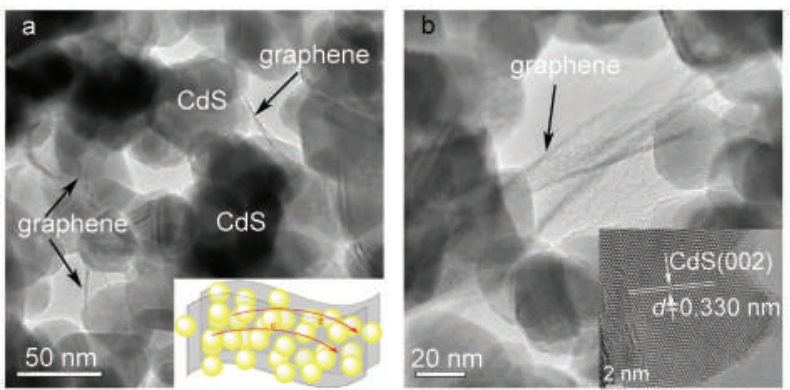

图 $3 \mathrm{CdS}$ /石墨烯复合材料的 TEM (a) 和 HRTEM (b)照片

Fig.3 TEM (a) and HRTEM (b) images of CdS/graphene composite

Insets in Fig. $3 \mathrm{a}$ and $3 \mathrm{~b}$ are the schematic structure of composite and the HRTEM image of CdS nanoparticles, respectively.
备的 CdS 为六方纤锌矿结构, 这与 HRTEM 的观察 结果相吻合. 各衍射峰强度较高, 说明样品晶体结 构完整，由 $\mathrm{CdS}(002)$ 晶面衍射峰的半峰宽，用谢乐 公式计算得到 $\mathrm{CdS}$ 的平均粒径为 $23.6 \mathrm{~nm}$, 这与 TEM 观察得到的结果基本一致. 不同石墨烯含量的 复合材料的 XRD 谱图与 $\mathrm{CdS}$ 基本一致, 说明在光催 化还原过程中 $\mathrm{CdS}$ 的晶型和粒径大小基本没有变 化. 此外, $\mathrm{CdS} /$ 石墨烯(1\%)的谱图中在 $2 \theta=23.48^{\circ}$ 处 出现了对应于石墨烯(002)晶面的衍射峰, 晶面间距 为 $0.38 \mathrm{~nm}$, 稍大于石墨粉的, 可能的原因是光催化 还原过程中氧化石墨烯的含氧基团未能完全还原， 同时说明复合材料的成功制备.

图 5 为氧化石墨、 $\mathrm{CdS}$ 和 $\mathrm{CdS} /$ 石 墨烯 $(1 \%)$ 复 合材料的红外光谱图. 氧化石墨分别在 $3396 \mathrm{~cm}^{-1}$ $\left(-\mathrm{OH}, \mathrm{H}_{2} \mathrm{O}\right) 、 1734 \mathrm{~cm}^{-1}(-\mathrm{C}=\mathrm{O}) 、 1622 \mathrm{~cm}^{-1}\left(\mathrm{H}_{2} \mathrm{O} /\right.$ $\mathrm{C}=\mathrm{C}) 、 1402 \mathrm{~cm}^{-1}(\mathrm{C}-\mathrm{O}) 、 1225 \mathrm{~cm}^{-1}(\mathrm{C}-\mathrm{O}-\mathrm{C})$ 和 $1051 \mathrm{~cm}^{-1}(\mathrm{C}-\mathrm{O})$ 处出现吸收峰, 说明氧化石墨含有 大量的含氧亲水基团. ${ }^{23,24}$ 复合材料的红外光谱与 CdS 相一致, 未出现对应于氧化石墨的特征吸收峰, 但 $\mathrm{CdS}$ 中对应 $-\mathrm{OH}\left(3449 \mathrm{~cm}^{-1}\right)$ 的吸收峰向低波数
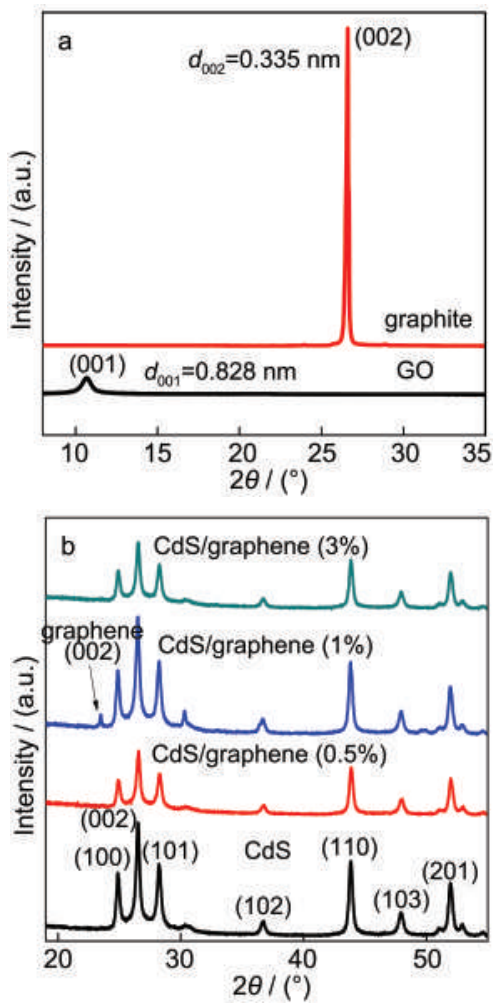

图 4 石墨和氧化石墨(GO) (a)以及 $\mathrm{CdS}$ 和 $\mathrm{CdS} /$ 石墨烯复 合材料(b)的 XRD 图谱

Fig.4 XRD patterns of graphite and graphite oxide (GO) (a), CdS and CdS/graphene composites (b)

$3 \%, 1 \%, 0.5 \%$ denote the mass fractions of graphene in the $\mathrm{CdS} /$ graphene composites. 


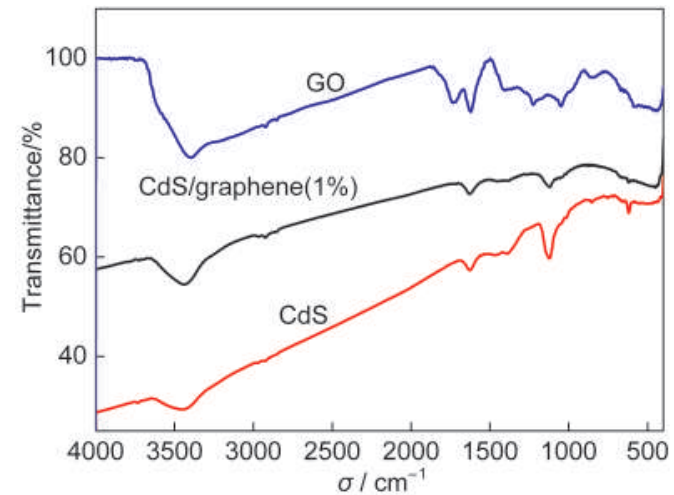

图 5 氧化石墨、CdS 和 $\mathrm{CdS} /$ 石墨烯 $(1 \%)$ 复合材料的 傅里叶变换红外光谱图

Fig.5 FTIR spectra of graphite oxide, $\mathrm{CdS}$, and CdS/graphene (1\%) composite

位移, 说明经光催化还原后氧化石墨烯转变为石墨 烯, 且 CdS 和石墨烯间存在较强的相互作用力.

图 6 给出了 $\mathrm{CdS}$ 和 $\mathrm{CdS} /$ 石墨烯复合材料中元素 的 XPS 谱图, 表 1 为样品中各元素的电子结合能、相 对摩尔百分含量以及 $\mathrm{Cd} / \mathrm{S}$ 和 $\mathrm{C} / \mathrm{O}$ 的摩尔比. 从图 6 和表 1 数据可以看出, $\mathrm{CdS}$ 和 $\mathrm{CdS} /$ 石墨烯复合材料表 面均含有 $\mathrm{Cd} 、 \mathrm{~S} 、 \mathrm{C}$ 和 $\mathrm{O}$ 元素, 无其它杂质, 样品纯度 高, 并且各样品中 $\mathrm{Cd} 3 d$ 和 $\mathrm{S} 2 p$ 的电子结合能 $\left(E_{\mathrm{B}}\right)$ 与 $\mathrm{CdS}$ 的文献值 ${ }^{25}$ 一致, 说明复合材料中存在 $\mathrm{CdS}$. 与 纯 $\mathrm{CdS}$ 相比, $\mathrm{CdS}$ /石墨烯复合材料中 C 元素含量均
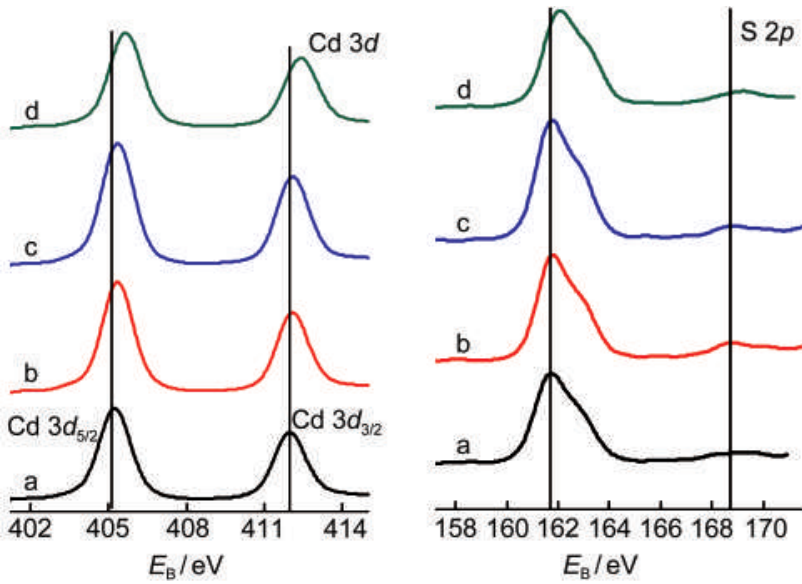

图 6 样品的 Cd 3d、S 2p、C 1s 和 O 1s 的 XPS 精细谱

Fig.6 High resolution Cd $3 d, \mathrm{~S} 2 p, \mathrm{C} 1 s$, and $\mathrm{O} 1 s$ XPS spectra of samples

(a) CdS, (b) CdS/graphene (0.5\%), (c) CdS/graphene (1\%), (d) CdS/graphene (3\%)

表 1 样品中各元素的结合能 $\left(E_{B}\right)$ 和相对摩尔百分含量 $(x)$

Table 1 Binding energy $\left(E_{\mathrm{B}}\right)$ and relative atomic percentage $(x)$ of various elements in different samples

\begin{tabular}{|c|c|c|c|c|c|c|c|c|c|c|}
\hline \multirow{2}{*}{ Sample } & \multicolumn{2}{|c|}{$\mathrm{Cd} 3 d$} & \multicolumn{2}{|c|}{$\mathrm{S} 2 p$} & \multirow{2}{*}{$x_{\mathrm{Cd}} / x_{\mathrm{S}}$} & \multicolumn{2}{|c|}{$\mathrm{C} 1 s$} & \multicolumn{2}{|c|}{$\mathrm{O} 1 s$} & \multirow{2}{*}{$x_{\mathrm{C}} / x_{\mathrm{O}}$} \\
\hline & $E_{\mathrm{B}} / \mathrm{eV}$ & $x_{\mathrm{Cd}} / \%$ & $E_{\mathrm{B}} / \mathrm{eV}$ & $x_{\mathrm{S}} / \%$ & & $E_{\mathrm{B}} / \mathrm{eV}$ & $x_{\mathrm{C}} / \%$ & $E_{\mathrm{B}} / \mathrm{eV}$ & $x_{\mathrm{O}} / \%$ & \\
\hline $\mathrm{CdS}$ & 405.24 & 27.66 & 161.74 & 18.59 & 1.49 & 285.00 & 27.98 & 531.64 & 25.77 & 1.09 \\
\hline CdS/graphene $(0.5 \%)$ & 405.38 & 26.60 & 161.82 & 16.41 & 1.65 & 285.00 & 28.65 & 531.82 & 28.35 & 1.01 \\
\hline CdS/graphene (1\%) & 405.38 & 30.15 & 161.76 & 19.31 & 1.56 & 285.00 & 29.81 & 531.88 & 20.91 & 1.43 \\
\hline CdS/graphene (3\%) & 405.68 & 23.68 & 162.08 & 15.16 & 1.56 & 285.00 & 36.88 & 532.18 & 24.28 & 1.52 \\
\hline
\end{tabular}


光生电子从 $\mathrm{CdS}$ 向石墨烯的快速迁移.?

\section{3 光电性能研究}

图 7 为纯 $\mathrm{CdS}$ 和不同石墨烯含量的 $\mathrm{CdS} /$ 石墨烯 复合光催化剂在可见光 $(\lambda \geq 420 \mathrm{~nm})$ 照射下的瞬态光 电流响应图. 从图中可以看出, 纯 $\mathrm{CdS}$ 电极在光照 射瞬间产生稳定、可逆的光电流, 但光电流较小, 约 为 $1.6 \mu \mathrm{A}$; 当引入 $0.5 \%$ 和 $1 \%$ 的石墨烯, $\mathrm{CdS} /$ 石墨烯 复合材料电极的光电流明显增强, 分别增大至 3.6 和 $3.4 \mu \mathrm{A}$, 相比纯 $\mathrm{CdS}$ 分别提高了约 1.3 和 1.1 倍, 且 光电流强度稳定; 当样品中石墨烯含量增至 3\%时, 光电流达到 $20.7 \mu \mathrm{A}$, 与纯 $\mathrm{CdS}$ 比较提高了近 12 倍, 但光电流强度随着光照时间的延长有所衰减, 这可 能与光生电子-空穴对在未完全还原的石墨烯片层 缺陷部位或残留的含氧基团处的复合有关. 以上结 果表明, 由于石墨烯良好的电子传递性能, 光激发 复合材料中 CdS 产生的电子可以通过石墨烯片层 迅速传递至 ITO 基体, 加快了电子在复合体系内的 迁移速率, 有效地抑制了光生电子-空穴对的复合, 从而提高了光生载流子的分离效率, 增强了复合材 料的光电转化性能. ${ }^{14-16}$

\section{4 光催化分解水产氢性能}

图 8 给出了 $\mathrm{CdS}$ 和不同石墨烯含量 $(w)$ 的 $\mathrm{CdS} /$ 石墨烯复合光催化剂在可见光照射下分解水产氢 的实验结果. 从图 $8 \mathrm{a}$ 可以看出, 未担载助催化剂 $\mathrm{Pt}$ 时, 纯 $\mathrm{CdS}$ 光催化产氢活性较低, 反应 $11 \mathrm{~h}$, 产氢量 约为 $0.43 \mu \mathrm{mol}$; 当引入 $0.5 \%$ 的石墨烯, 相同反应条 件下复合材料光催化产氢量为 $2.57 \mu \mathrm{mol}$, 比纯 CdS 提高了约 5 倍, 当石墨烯含量进一步增加(1\%), 产氢

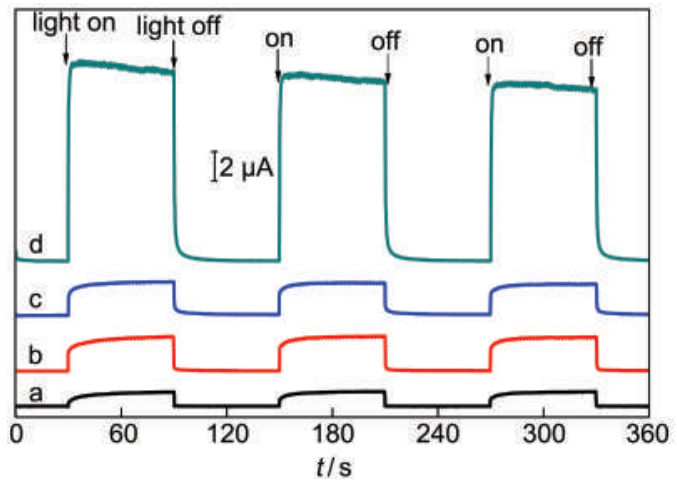

图 $7 \mathrm{CdS}$ 和 $\mathrm{CdS} /$ 石墨烯复合材料在可见光照射下 $(\lambda \geq 420 \mathrm{~nm})$ 的瞬态光电流曲线

Fig.7 Transient photocurrent profiles of $\mathrm{CdS}$ and CdS/graphene composites under visible light irradiation $(\lambda \geq \mathbf{4 2 0} \mathbf{~ n m})$

(a) CdS, (b) $\mathrm{CdS} /$ graphene $(0.5 \%)$, (c) $\mathrm{CdS} /$ graphene (1\%), (d) $\mathrm{CdS} /$ graphene $(3 \%)$
活性有所降低 $(0.77 \mu \mathrm{mol})$, 但仍高于纯 $\mathrm{CdS}$; 当石墨 烯含量为 $3 \%$ 时, 产氢活性 $(0.27 \mu \mathrm{mol})$ 低于 $\mathrm{CdS}$ 样品 本身. 图 $8 \mathrm{~b}$ 的结果表明, 当光催化原位担载 $0.5 \%$ 的 $\mathrm{Pt}$ 之后, $\mathrm{CdS}$ 和 $\mathrm{CdS} /$ 石墨烯复合光催化剂的光催化 分解水产氢活性进一步增强, 与未担载相比提高了 2 个数量级以上. 在所研究的石墨烯含量范围内, $\mathrm{CdS} /$ 石墨烯复合光催化剂的活性均高于纯 $\mathrm{CdS}$, 且 随着石墨烯含量的增加, 反应 $10 \mathrm{~h}$, 产氢量先增大后 减小, 当石墨烯含量为 $1 \%$ 时, 产氢量达到 56.4 $\mu \mathrm{mol}$, 高于纯 $\mathrm{CdS}$ 约 1.5 倍 $(22.9 \mu \mathrm{mol})$. 以上结果表 明, 少量石墨烯 $(0.5 \%-1 \%)$ 的引入即可明显提高复 合体系光生电荷的分离效率, 增强 $\mathrm{CdS}$ 的光催化活 性; 而当石墨烯含量过大时 $(>3 \%)$, 则可能会降低 $\mathrm{CdS}$ 纳米粒子的有效光照面积, 导致光催化活性降 低. ${ }^{9}$ 研究表明, $\mathrm{CdS}$ 的禁带宽度约为 $2.4 \mathrm{eV}$, 导带电 子和价带空穴相对于标准氢电极的电极电势分别 为 -0.52 和 $1.88 \mathrm{~V}$, 根据公式: $E(\mathrm{eV})=-4.5-E_{\mathrm{NHE}}(\mathrm{V}),{ }^{27}$ 计算得到相应的功函数分别为 -3.98 和 $-6.38 \mathrm{eV}$, 而 石墨烯和 $\mathrm{Pt}$ 的功函数为 -4.42 和 $-5.65 \mathrm{eV}^{7} \mathrm{CdS} /$ 石 墨烯复合材料可见光催化分解水产氢反应机理如
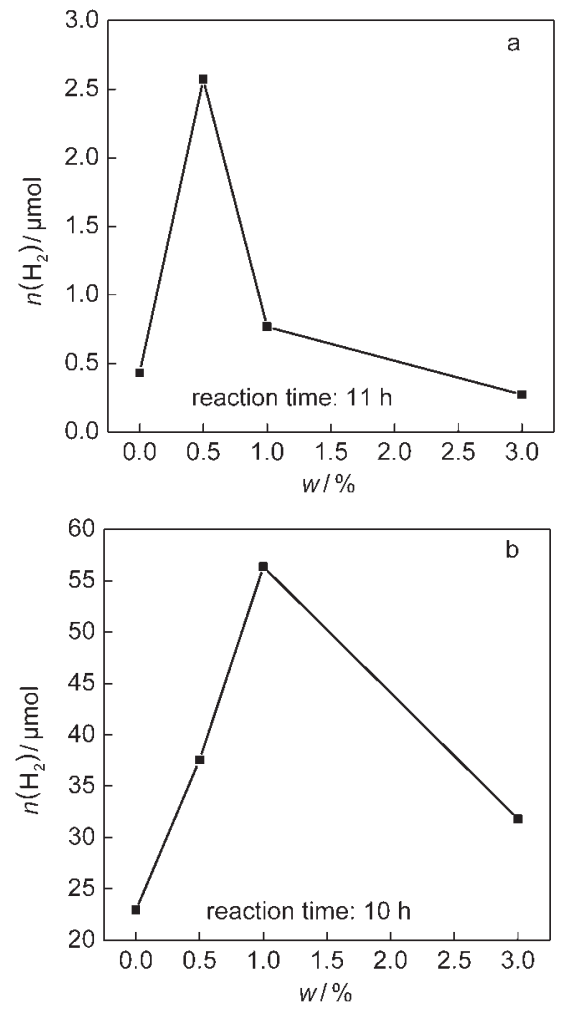

图 $8 \mathrm{CdS}$ 和 $\mathrm{CdS} /$ 石墨烯 $(w)$ 复合材料可见光催化 分解水产氢性能

Fig.8 Photocatalytic activities for hydrogen evolution over $\mathrm{CdS}$ and $\mathrm{CdS} /$ graphene $(w)$ composites

(a) without Pt loading, (b) with $0.5 \%$ Pt loading 


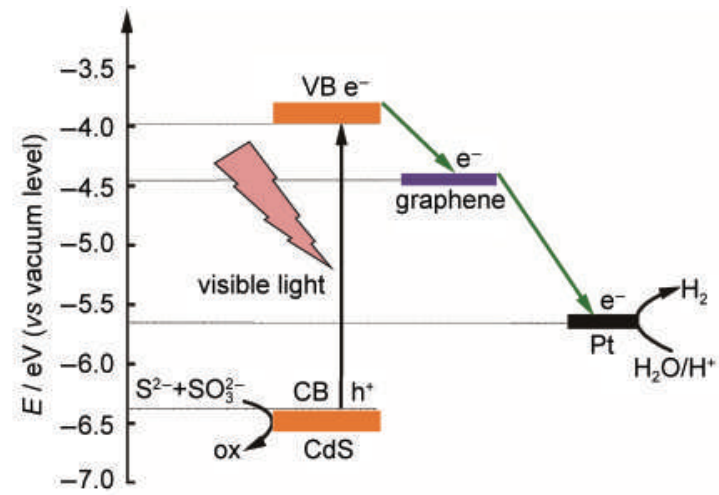

图 $9 \mathrm{CdS} /$ 石墨烯复合材料可见光下光催化分解 水产氢的能级图

Fig.9 Energy level diagram for hydrogen evolution over CdS/graphene composite photocatalyst

图 9 所示. 由上面的分析可知, 石墨烯的费米能级低 于 $\mathrm{CdS}$ 导带电子的, 因此光激发 $\mathrm{CdS}$ 产生的导带电 子可较易地迁移至石墨烯上，而石墨烯是优异的电 子受体和传递介质, 可加快光生电子向表面的迁移 速率, 有效地降低了光生电子和空穴的复合, 提高 了载流子的分离效率, ${ }^{7} \mathrm{Pt}$ 的担载可进一步促进载 流子的分离和降低析氢过电位, ${ }^{28,29}$ 从而提高复合材 料的光催化分解水产氢的效率.

\section{4 结 论}

采用光催化还原法成功制备了 $\mathrm{CdS}$ /石墨烯复 合光催化材料. 石墨烯穿插于 $\mathrm{CdS}$ 纳米粒子之间, 并与 $\mathrm{CdS}$ 之间以较强的作用力结合在一起. 复合材 料中石墨烯优异的电子传输性能加快了 $\mathrm{CdS}$ 光生 电子的迁移速率, 抑制了光生载流子的复合. 与 $\mathrm{CdS}$ 相比, $\mathrm{CdS} /$ 石墨烯复合材料在可见光下具有更为优 异光电性能和光催化分解水产氢性能, 石墨烯含量 对复合材料的性能有较大的影响.

\section{References}

(1) Novoselov, K. S.; Geim, A. K.; Morozov, S. V.; Jiang, D.; Zhang, Y.; Dubonos, S. V.; Grigorieva, I. V.; Firsov, A. A. Science 2004, 306, 666.

(2) Geim, A. K.; Novoselov, K. S. Nat. Mat. 2007, 6, 183.

(3) Stoller, M. D.; Park, S.; Zhu, Y. W.; An, J.; Ruoff, R. S. Nano Lett. 2008, 8, 3498 .

(4) Bolotin, K. I.; Sikes, K. J.; Jiang, Z.; Klima, M.; Fudenberg, G.; Hone, J.; Kim, P.; Stormer, H. L. Solid State Commun. 2008, 146,351

(5) Geng, X. M.; Niu, L.; Xing, Z. Y.; Song, R. S.; Liu, G. T.; Sun, M. T.; Cheng, G. S.; Zhong, H. J.; Liu, Z. H.; Zhang, Z. J.; Sun, L. F.; Xu, H. X.; Lu, L.; Liu, L. W. Adv. Mater. 2010, 22, 638.

(6) Lin, Y.; Zhang, K.; Chen, W. F.; Liu, Y. D.; Geng, Z, G.; Zeng,
J.; Pan, N.; Yan, L. F.; Wang, X. P.; Hou, J. G. ACS Nano 2010, 4, 3033.

(7) Guo, C. X.; Yang, H. B.; Sheng, Z. M.; Lu, Z. S.; Song, Q. L.; Li, C. M. Angew. Chem. Int. Edit. 2010, 49, 3014.

(8) Kim, S. R.; Parvez, M. K.; Chhowalla, M. Chem. Phys. Lett. 2009, 483, 124.

(9) Zhang, Y. H.; Tang, Z. R.; Fu, X. Z.; Xu, Y. J. ACS Nano 2010, 4, 7303.

(10) Du, J.; Zhang, H.; Lv, X. J.; Li, Y. M.; Wang, Y.; Li, J. H. ACS Nano 2010, 4, 380.

(11) Akhavan, O.; Ghaderi, E. J. Phys. Chem. C 2009, 113, 20214.

(12) Chen, C.; Cai, W. M.; Long, M. C.; Zhou, B. X.; Wu, Y. H.; Wu, D. Y.; Feng, Y. J. ACS Nano 2010, 4, 6425.

(13) Xu, T. G.; Zhang, L. W.; Cheng, H. Y.; Zhu, Y. F. Appl. Catal. B 2011, 101, 382.

(14) Lightcap, I. V.; Kosel, T. H., Kamat, P. V. Nano Lett. 2010, 10, 577.

(15) Ng, Y. H.; Lightcap, I. V.; Goodwin, K.; Matsumura, M.; Kamat, P. V. J. Phys. Chem. Lett. 2010, 1, 2222.

(16) Kamat, P. V. J. Phys. Chem. Lett. 2010, 1, 520.

(17) Zhang, X. Y.; Li, H. P.; Cui, X. L.; Lin, Y. H. J. Mater. Chem. 2010, 20, 2801.

(18) Ng, Y. H.; Iwase, A.; Kudo, A.; Amal, R. J. Phys. Chem. Lett. 2010, 1, 2607.

(19) Hummers, W. S.; Offeman, R. E. J. Am. Chem. Soc. 1958, 80, 1339.

(20) Kovtyukhova, N. I.; Ollivier, P. J.; Martin, B. R.; Mallouk, T. E.; Chizhik, S. A.; Buzaneva, E. V.; Gorchinskiy, A. D. Chem. Mater. 1999, 11, 771.

(21) Yan, H. J.; Yang, J. H.; Ma, G. J.; Wu, G. P.; Zong, X.; Lei, Z. B.; Shi, J. Y.; Li, C. J. Catal. 2009, 266, 165.

(22) Yang, Y. H.; Sun, H. J.; Peng, T. J.; Huang, Q. Acta Phys. -Chim. Sin. 2011, 27, 736. [杨勇辉, 孙红娟, 彭同江, 黄 桥. 物理化 学学报, 2011, 27, 736.]

(23) Szabó, T.; Berkesi, O.; Forgó, P.; Josepovits, K.; Sanakis, Y.; Petridis, D.; Dékány, I. Chem. Mater. 2006, 18, 2740.

(24) Lee, D. W.; De Los Santos, V. L.; Seo, J. W.; Felix, L. L.; Bustamante, D. A.; Cole, J. M.; Barnes, C. H. W. J. Phys. Chem. $B$ 2010, 114, 5723.

(25) Xi, Y. Y.; Zhou, J. Z.; Zhang, Y.; Dong, P.; Cai, C. D.; Huang, H. G.; Lin, Z. H. Chem. J. Chin. Univ. 1999, 20, 937 . [席燕燕, 周剑章, 张 彦, 董 平, 蔡成东, 黄怀国, 林仲华. 高等学校化 学学报, 1999, 20, 937.]

(26) Zhang, H. Q.; Chen, K. X.; Jin, Z. S. Chin. J. Appl. Chem. 1999, 14, 98. [张虎勤, 陈开勋, 金振声. 应用化学, 1999, 14, 98.]

(27) Xiong, Z. G.; Zhang, L. L.; Ma, J. Z.; Zhao, X. S. Chem. Commun. 2010, 46, 6099.

(28) Jin, Z. L.; Lü, G. X. J. Mol. Catal. (China) 2004, 18, 310. [靳治良, 吕功煊. 分子催化, 2004, 18, 310.]

(29) Zhang, X. J.; Tang, C. Q.; Jin, Z. L.; Lü, G. X.; Li, S. B. Acta Phys. -Chim. Sin. 2011, 27, 1143. [张晓杰, 汤长青, 靳治良, 吕功煊, 李树本. 物理化学学报, 2011, 27, 1143.] 